\title{
Immersive flight for surveillance applications
}

\author{
Xavier Righetti ${ }^{\star}$, Sylvain Cardin ${ }^{\dagger}$, Daniel Thalmann ${ }^{\ddagger}$, Frederic Vexo ${ }^{\S}$ \\ EPFL, VRlab, Switzerland
}

\begin{abstract}
In this paper, we present a VR-based first-person view paradigm applied to a tele-surveillance application. Using an Unmanned Air Vehicle (UAV), we have developed an intuitive tangible interface between the pilot and his airship (blimp). The idea is to make transparent the manipulation of an embedded camera by controlling it instinctively with the head's movement so that the user is available for other tasks such as piloting the blimp. IN other words, the user becomes part of the interface. Using the same paradigm for sensing real-time acquired sensor data, a vibro-tactile belt worn by the user will indicate the resistance offered by the wind and thus will increase the feeling of telepresence. The results of our experience show that our system is reliable and enhances the situational awareness of the pilot.
\end{abstract}

CR Categories: I.3.7 Three-Dimensional Graphics and Realism Virtual Reality

Keywords: Telepresence, tele-operation, Head-Mounted Display, surveillance, haptic interface.

\section{INTRODUCTION}

Aerial surveillance is a fast-growing topic in the commercial and research field. Indeed, many "security sensitive" events such as olympic games are now fully monitored by drones, helicopters or blimps. Aerial surveillance offers the best point of view to ensure maximum visibility while giving an outline of the general situation. Other possible applications include search and rescue operations, environmental surveillance and modeling and traffic monitoring. Furthermore, compared to airplanes, blimps are able to hover in a stationary position, which is a primordial capability for many monitoring applications. They produce very low noise, turbulences and internal vibrations, generate low operational costs and have a long endurance. In addition, blimps are the safest UAVs: even if the blimp is perforated, it doesn't represent a potential danger for the surrounding people. Thus, blimps seem to be the perfect platform for aerial observation. In many cases, monitoring missions require the blimp to carry at least a pilot and a copilot. Hence, it has a substantial incidence on the fixed and operational costs. For these reasons, we have opted for an unmanned airship, entirely controlled by only one person on the ground.

In this paper, blimps are considered as a natural extension of our selves. They let us wander and observe in a first-person view.

\footnotetext{
* e-mail: Xavier.Righetti@epfl.ch

$\dagger$ e-mail: Sylvain.Cardin@epfl.ch

† e-mail: Daniel.Thalmann@epfl.ch

§ e-mail: Frederic.Vexo@epfl.ch
}

IEEE Symposium on 3D User Interfaces 2007 March 10 - 11, Charlotte, North Carolina, USA $1-4244-0907-1 / 07 / \$ 25.00$ @2007 IEEE

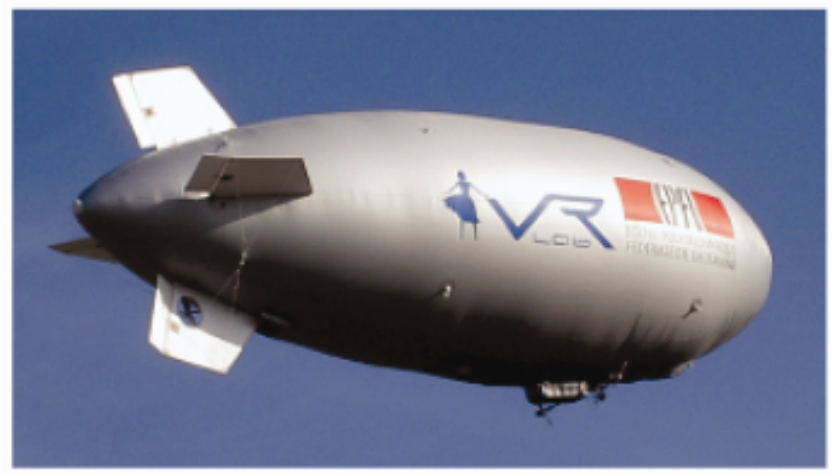

Figure 1: VRLab's Blimp.

Moreover, this increasing amount of input and output data passing from the blimp to the user urges us to elaborate new custom interfaces of communication so that no information may be neglected or misunderstood. This is especially true in our case where we often deal with delicate operations such as the piloting of the blimp without direct visual contact.

Next chapter will review previous works published in this field of research. Our system will be tackled in Section 3 by describing the interaction paradigms.

\section{PREVIOUS WORKS}

In [3], Alberto Elfes et al. categorize the differences between airplanes, airships and helicopters for environmental research and monitoring missions. Their results highlights that blimps are better under all the aspects, except for the manoeuvrability. Besides, if the blimp is perforated or its control system crashes, it will land slowly on the ground with almost no risk of hurting anyone. This would definitely not be the case for a RC helicopter, for example. Since the applicability of our research is observation and surveillance, we thus have opted for the use of an airship (blimp).

In [5], Lacroix et al. propose an approach in which they use an unmanned autonomous blimp to model the environment using low altitude stereoscopic imagery. The on-board sensors consist of a stereoscopic b\&w camera, a differential GPS receiver, a compass and a wind sensor. As for the CPU, they added an EBX form factor motherboard that includes 3 firewire ports amongst other things. Because of energy consumption concerns, we have opted for a much less powerful CPU: a PIC18 microcontroller. The second reason of this choice comes from the size of our blimp, which is significantly smaller and thus has a lower payload. Also, for safety purposes, they envisaged a radio controlled switch so that an operator can retrieve the control of the blimp at any time in case of mechanical or electronic failure of the blimp. This element will also be integrated in our prototype. For their experiment, Lacroix and al. decided to replace the two thrusters batteries with Lithium Ion Polymer (LiPo) batteries, that have a much more interesting power/weight ratio. The same kind of batteries are used for our prototype.

In [7], the authors have shown that the teleoperation of a vehicle using haptic devices is more efficient when having a gesture interface. 
In [6], Ott and al. presented a surveillance and security system that exploits advanced VR technologies. Indeed, they encouraged the use of a novel interface based on eye-tracking technologies which minimizes the use of keyboards and other input devices. Furthermore, they used an Immersion Haptic Workstation ${ }^{\mathrm{TM}}$ as a remote control for the blimp. Right hand was used to control the aerodynamic stabilizer (by moving the hand forward/backward and left/right), whereas left hand was used to control the engine power. During the experiment, the blimp crashed heavily because the pilot did not take into consideration a sudden relatively strong localized wind.

\section{OUR SYSTEM}

To pilot a blimp is a quite difficult task, especially when the pilot has to accomplish an observation mission at the same time. But this can be achieved if three mandatory conditions are met: the interface must be accurate, instructive and intuitive. In other words, the pilot has to have an accurate natural feedback of what the blimp sees and feels in real-time. The faster he understands the situation, the faster he will react accordingly.

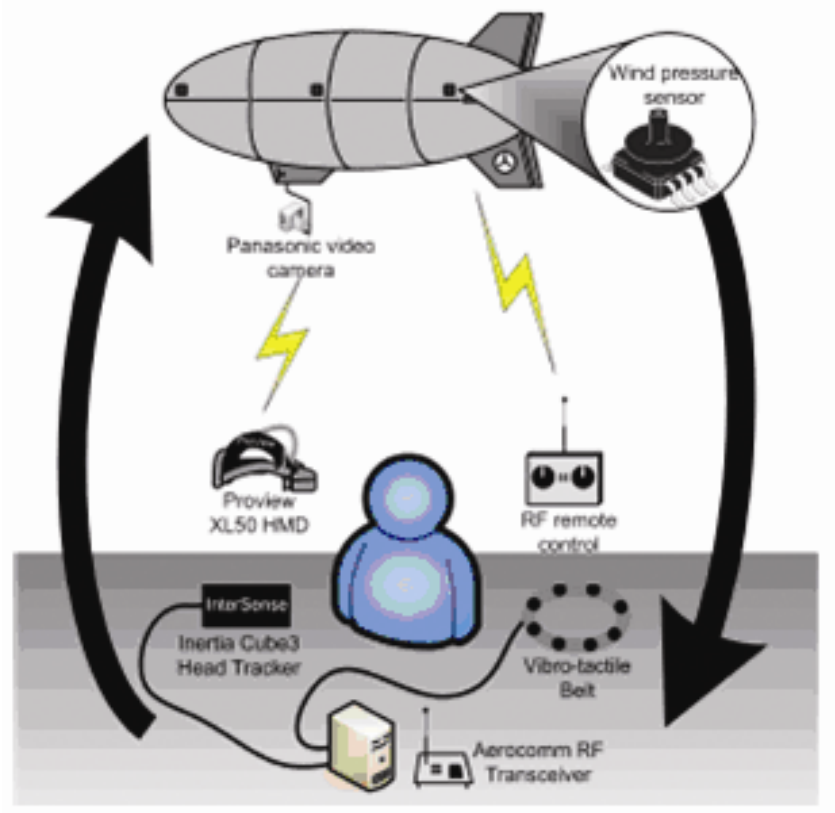

Figure 2: Overall Architecture of our system

In order to get a system which fulfils our main objective, we have set four basic constraints. First, the blimp must be operated by one single person on the ground. Second, it must be able to fly for at least one hour non-stop. Third, its operating range must be at least $2 \mathrm{~km}$. And fourth it must be able to fly in light wind conditions (less than $30 \mathrm{~km} / \mathrm{h}$ ).

Wind is a very crucial factor for the piloting of a blimp. Even the smallest breeze will have an effect on the blimp.This is why we implemented a vibro- tactile belt, that intuitively indicates the direction of the wind as well as its strength.

Consequently we have stressed a natural and efficient haptic interface integrating a Head-Mounted Display (HMD), a vibrotactile belt and a pair of headphones. The live video feed taken from the onboard video camera is displayed on the HMD. Head movements are tracked and replicated to the video camera thanks to a two-axis robotic arm holding the camera. The response time should be kept less than $40 \mathrm{~ms}$ which corresponds to $25 \mathrm{fps}$. As shown on Figure 2, our system uses three distinct wireless connections. One for the video feed, one for getting and sending informations trough the RF transceiver to the embedded microcontroller, and finally, one for piloting the blimp using a classical analog RF remote control.

\subsection{R/C Blimp}

The blimp we have used for several teleoperation experiments measures 6.75 meters long by 2.20 meters width. This unmanned air vehicle is filled with $11 \mathrm{~m} 3$ of Helium gas. Its tare weight is $7 \mathrm{~kg}$ and its maximum payload is $2 \mathrm{~kg}$ for all onboard equipment. Two electric Greman brushless motors propel the blimp at $35 \mathrm{~km} / \mathrm{h}$ in flat wind conditions. Each motor has 15 Newton of power and are plugged to a $6600 \mathrm{mAh}$ LiPo battery which allows a 1 hour continuous flight at half speed.

\subsection{On-board Equipment}

- Microcontroller: We have opted for a PIC 18F6720 from Microchip: a basic microcontroller with very low power consumption. It was programmed using a combination of assembler and standard ANSI C. A single PIC18 acts as the central processing unit. It gathers data from embedded sensors, controls on-board actuators and communicates to the on-board modem via an RS-232 serial link. The PIC18 has several builtin Pulse-Width Modulation (PWM) output, but we have created our own PWM generation method, which can drive simultaneously up to 16 servos.

- Video Camera: We have used a Panasonic 3CCD video camera for tele-observation. The video camera weights $1.2 \mathrm{~kg}$ and has a $12 \mathrm{x}$ optical zoom. The zooming capability is very important to us, because as a surveillance system, we want to be able to get a very detailed video feed. The video camera is mounted on a two axis (pan / tilt) robotic arm which gives us a field of view of more than 180 degrees on both axis. The video feed is sent using a proprietary analog wireless transmission system, that can range up to 2 kilometers without obstacles. The video camera is powered with its own battery and thus can record non-stop for approximately 2 hours, which is more than enough.

- Wind Sensors: As we have stated earlier, wind is a very dangerous factor of which account should be held. Therefore we have mapped its behavior on a vibro-tactile belt used in our previous researches $[1,2]$. We have embedded six wind sensors in order to measure the speed and orientation of the relative wind in the longitudinal plane. The MPXV5004GC7U piezoresistive transducer (shown on Figure 7) provides an accurate, high level analog output signal that is proportional to the applied pressure.

- Servomechanism: A major limitation arising from the use of an embedded video camera for monitoring applications is the relatively small field of view offered by the lens of the video camera. To solve the problem, mounted the camera on a robotic arm in order to control the pan and tilt and thus to have a 180 degrees view.. Consequently, we have used three Graupner servos for controlling the embedded camera. Each of our three servos have their own characteristics, as their function differs. The first and the second servos are used for controlling the pan and tilt of the camera while the third one enables the optical zooming functionality. Servomechanisms are controlled by sending them a PWM signal. The period of the signal must be $20 \mathrm{~ms}$. A pulse is emitted at the beginning of the period. The position of the servo varies in fonction of the length of the pulse. 
- Modem: Information gathered from on-board sensors is processed by the microcontroller and then transmitted to the ground via Aerocomm's RF transceiver. On the opposite, movements detected by the head tracker use the same RF connection to reach the embedded microcontroller. The two modems can communicate up to 115200 bps but have been set to $57600 \mathrm{bps}$ in our system. The AC4486 transceivers provide an asynchronous TTL/RS-485 level serial interface.

\subsection{Embedded Software}

The code has been programmed and assembled using PCWH Compiler from Custom Computer Services, Inc. This third party software lets the programmer code in standard ANSI C. In our software architecture, the PIC18 microcontroller is $100 \%$ interrupt-driven. It is a commonly used technique for real-time computing.

\subsubsection{Serial Communication}

We have developed a simple message-based communication system relying on the RS-232 serial connection. The communication has been established at $57600 \mathrm{bps}$ between the embedded PIC microcontroller and the computer located on the ground. This communication link is used to transmit information from the wind sensors to the vibro-tactile belt worn by the user and from the head tracking device to the robotic arm supporting the video camera.

Each time the microcontroller receives a message, an interrupt occurs and the message is treated according to its type. A checksum verification method has been integrated in the messaging system, so that the microcontroller knows which packet is corrupted and thus can ask for the same packet again. If the message length is misunderstood, for example $0 \times 3 \mathrm{~F}$ instead of $0 \times 2 F$, the microcontroller will have to wait for 16 inexistant bytes, thus resulting in excessively long waiting times and corrupted PWM signals. This is why the checksum is calculated only from the type and the size of the message.

\subsubsection{PWM Generation}

Servos are driven by a modulated signal called Pulse Width Modulation. In our case, thePWMis controlled using the built-in Capture / Compare (CCP1) instruction. In short, the microcontroller generates a chained list which contains the physical address of the servo and the accurate duration of the pulse expressed in cycles. Every 50'027 cycles (equivalent to $20 \mathrm{~ms}$.), the chained list is traversed and the position of the 16 servos are updated.

\subsection{Ground Equipment}

Ground Equipment let us interact with the distant blimp. It is composed of a PC, a Head-Mounted Display (HMD) with headphones, and Head tracking device and a vibro-tactile belt.

- Personal Computer: we have developed a simple software interface in $\mathrm{C}++$ to be able to get real-time values given by the IntertiaCube 3 head tracker. The software automatically sends these new values to the microcontroller via the modem.

- HMD: The PLM-S700E offers a virtual experience of watching a 30 inch screen viewed from 1.2 meters away when connected to a personal computer. In addition, built-in stereo headphones provide quality sound. The display unit is compact in size and can be folded for portability. Furthermore, the lightweight display unit (120g.) provides an easy fit to the head and ensures comfort over extended hours of use, even for users who wear eye glasses. Sony's Glasstron has two 0.7" LCDs capable of displaying 1.55 million pixels.

- InertiaCube3 Head tracker: In order to map the head movements to the camera, the HMD is tracked using the InertiaCube3, an orientation tracker commercialized by Intersense. This tracker is very accurate and has a refresh rate of $180 \mathrm{~Hz}$ and a minimum latency of $2 \mathrm{~ms}$. It has three degrees of freedom (Yaw, Pitch and Roll) with an angular range of 360 degrees on all axes and thus is more than capable of tracking the head's movements, but we will use only the Yaw and Pitch information.

- Vibro-tactile Belt; Since the early sixties, researches have been conducted on vibrotactile stimulation in term of amplitude, frequency and spatial cues. The results of those studies have shown that we are able to distinguish up to nine levels of vibration [4]. Also, vibrating devices such as our vibro-tactile belt have already been proved useful to enhance navigation ability. For example, J. Van Erp et al. [8] conducted a research on vibrating belts for navigation waypoint enhancement. They concluded that mapping waypoint direction on the location of vibration is an intuitive and effective coding scheme. Our device consists in a belt in which we embedded 8 equally placed vibrators. A calibration system has been implemented so that each vibrators can be tuned individually or globally.

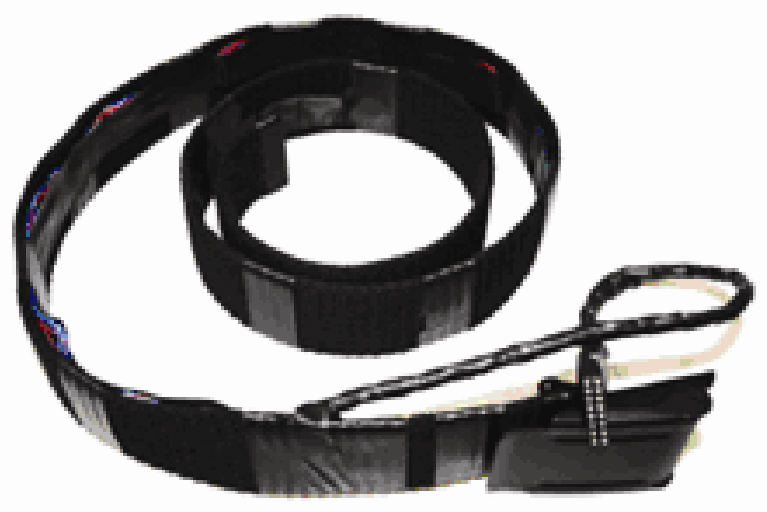

Figure 3: The vibro-tactile belt developed at VRLab

\subsection{User Interface}

The goal of our tangible interface is to improve the flying conditions. Since the user must be able to pilot the blimp anywhere in the three dimensions, he needs complete awareness of the situation. Thus, the 3D input device (head tracker) will replicate the physical movements of the head while the 3D output device (vibro-tactile belt) will replicate the movements of the wind. The user interface can be divided in three categories: what you see, what you feel and what you hear.

- Vision System: The camera system used for telepresence is a two-degree of freedom device that can pitch and yaw according to the user's head orientation. The orientation of the head is calculated using the InertiaCube 3 head tracker attached to the HMD. In addition, the analog RF remote control has two optional buttons used by the pilot to control the zoom of the embedded video camera.

- Haptic System: Vibrating actuators placed on a belt [1] will unconsciously inform us of the resistance of the wind. The location of the active vibrator on the body will give us of the direction of the wind while the strength of the vibration will be 
proportioned to the strength of the wind. As mentioned earlier, wind is a critical factor for a lighter than air vehicle. It is very important for the pilot to be aware of this variable in real time. Hence the vibro-tactile belt ensures this kind of permanent contact.

- Auditory System: Because we don't need to hear what is happening around the blimp, this leaves us a blank canal for passing information to the user. Compared to visual display, it is harder to ignore what you hear. Thus, critical information such as the distance in meters of obstacles detected by embedded sonar sensors, the altitude, the signal strength and the low battery level will be communicated to the user through headphones.

\section{PRELIMINARY TESTS \& RESULTS}

First tests have been conducted inside the lab without using the blimp. The users were sitting in a room next to a computer while the video camera mounted on the robotic arm had been attached to the ceiling of a close room. They interacted with the camera one by one by wearing the HMD with the head tracking device mounted on top of it.

Our tele-observation method proved to be very satisfying as it really gave an natural immersion feeling. During the experiment, we have realized that in order to efficiently drive several servos using the internal timer of the PIC18 microcontroller, we needed a higher resolution. Therefore we changed from a 8 bit internal timer to a 16bit internal timer which overflows every 65535 (0xFFFF) cycles.

Additionally, if two servos have a very close position, the algorithm will remove the delta between them and thus force the two servos at the exact same position. This operation ensures that the microcontroller is not missing a servo during execution time. In order to know specific execution times, we have implemented a precision verification routines to highlight the number of cycles of delay. It showed us that a simple operation such as an interruption assignment takes more than 10 cycles to be executed. It means that the value of our delta shouldn't be less than 10, otherwise at the time of the interruption, the value of the timer will already be greater than the value of the interruption and thus the microcontroller will not drive the servo at all.

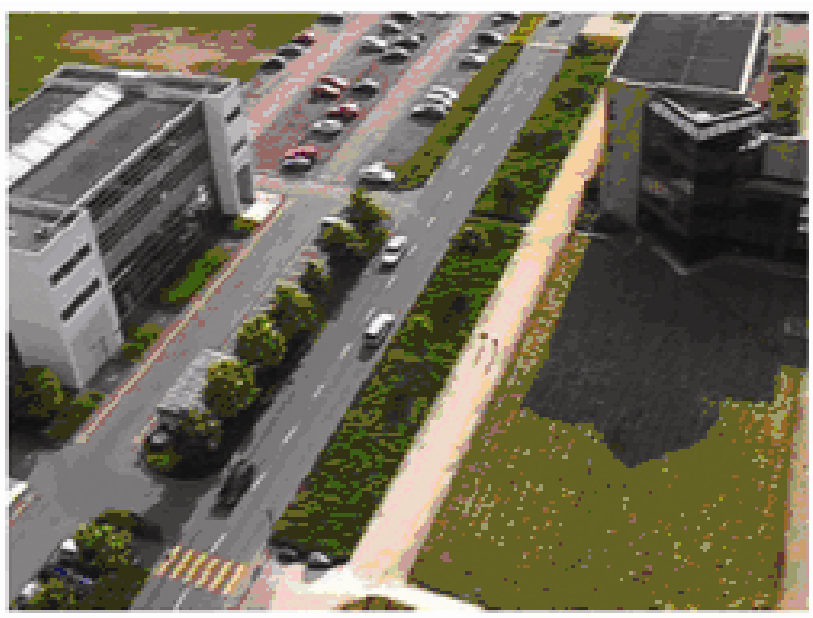

Figure 4: Aerial snapshot taken from the embedded video camera
For the second experience, we have tested the blimp on the campus of the EPFL. For this experiment, 5 researchers from our lab had to pilot the blimp for 10 minutes. Their mission was to fly over an outdoor parking without crashing the blimp and at the same time to count the parked cars. Prior to the experiment, participating users had been trained for 20 minutes using the simulation programmed named Xplane.

At the end of the experiment, four out of five participants managed to correctly count the cars. None of them crashed the blimp, but they all had some trouble for landing the blimp in a precise spot. The problem was the difficulty to make the blimp go down and at the same time direct it to the landing spot. Therefore we added a little ballast so that it gently comes down by itself. This ballast has been proved useful for landing approaches but also when our blimp's embedded microcontroller crashed. Indeed, we wouldn't want to loose our search \& rescue tool.

\section{FUTURE WORK}

Our next step is to be able to control the blimp using a digital input, such as a joystick. Moreover, next step is to integrate an embedded video processing software that would let us lock an object on the ground such as a car or a person, so that the blimp and its video camera would constantly follow it. This would be done in order to let the pilot fully concentrate on the observation task. For safety reasons, if somehow the communication fails or the microcontroller crashes, it would be wise to add another backup communication channel. This requirement will be met by adding an emergency switch on the blimp that can be teleactivated by a classical RF controller in order to regain control.

\section{ACKNOWLEDGEMENTS}

This research has been partially funded by the EC in the framework of the European Network of Excellence ISTINTUITION (http://www.intuition-eunetwork.net).

\section{REFERENCES}

[1] S. Cardin, F. Vexo, D. Thalmann. Wearable System for Mobility Improvement of Visually Impaired People. Visual computer journal, 2007 (to appear).

[2] S. Cardin, F. Vexo, D. Thalmann. Vibro-Tactile Interface for Enhancing Piloting Abilities During Long Term Flight. Journal of Robotics and Mechatronics, 18(3), 2006.

[3] A. Elfes, S. Bergerman, M. Ramos. A semi-autonomous robotic airship for environmental monitoring missions. Robotics and Automation, 1998. Proceedings. 1998 IEEE International Conference on, 4, 1998.

[4] G. D. Goff. Differential discrimination of frequency of cutaneous mechanical vibration. Journal of Experimental Psychology, 74:294299, 1967

[5] S. Lacroix, I. Jung. High resolution terrain mapping with an autonomous blimp. Intelligent Robots and System, 2002. IEEE/RSJ International Conference on, 1, 2002.

[6] R. Ott, M. Gutierrez, D. Thalmann, and F. Vexo. Advanced Virtual Reality Technologies for Surveillance and Security Applications. Proceedings of the ACM SIGGRAPH International Conference on Virtual Reality Continuum and Its Applications (VRCIA 2006), Hong-Kong, 14-17 June 2006.

[7] R. Ott, M. Gutierrez, D. Thalmann, and F. Vexo. VR Haptic Interfaces for Teleoperation: an Evaluation Study. Proceedings of the IEEE Intelligent Vehicles Symposium: IV'05, Las Vegas, Nevada, USA, pages 788-793, 6-8 June 2005.

[8] J. Van Erp, H. Van Veen, C. Janseen, and D. T. Vibrotactile Waypoint Navigation at Sea and in the Air: two Case study. ACM Transactions on Applied Perception, 2005. 